\title{
How Web Tools Affect Efficiency and Effectiveness of Teachers and Learners
}

\author{
John Rosbottom \\ University of Portsmouth \\ Portsmouth, United Kingdom
}

\author{
Jean-Marc Lecarpentier \\ Université de Caen \\ Caen, France
}

\begin{abstract}
Internet and web-based tools are essential in eLearning. They can also act as a supplement to learning in campus-based environments. Such tools can bring great benefits to a teacher, or they can generate considerable overhead work resulting in a loss of efficiency and effectiveness. We identify some principles, and some practices, for enhancing the efficiency and effectiveness of teachers using webbased, e-Learning and e-Administration tools in a mostly campus-based learning environment. The key principles we seek in e-Learning and $e$ Administration tools are simplicity, versatility and a decentralized, but collaborative, mode of operation which we illustrate through case studies of our own work and that of others. We review relevant learning and assessment models, explicate the key principles for supporting the teacher's role with web-based tools, and provide numerous illustrative examples.
\end{abstract}

\section{Introduction}

Like most Higher Education Organisations, the Universities of Portsmouth and Caen have set up Virtual Learning Environments (VLE) for all teachers and students. Some claim that the VLE is redundant nowadays because its functions can be achieved more easily and often more effectively using readily available web-based software. As users of Web2.0 tools and of Open Source Software we were not always satisfied by the tools provided by our respective organisations and started creating and setting up our own, using web-tools already available or creating new ones.

Students at the Département d'Informatique are typically from 3rd to 5th year students, with some students completely new to the domain. Students at the School of Computing in Portsmouth have a similar profile, although they are typically younger. Therefore IT skills vary greatly, with some students already using web tools like twitter, facebook, email and delicious every day and others discovering the potential of the web. As an organisation, the Département d'Informatique uses Open Source Software wherever possible and strongly encourages teachers and students to do so as well. In this paper we consider what, if any, gains in effectiveness and efficiency we can obtain through e-Learning and present the tools used as well as the benefits and drawbacks the authors have experienced.

\section{Efficiency and effectiveness}

\subsection{Conceptual Analysis}

To be effective means to carry out tasks successfully. To be efficient means to carry out tasks with few or zero errors, and with optimum speed, maximising the success while minimising the work required to achieve success. How can these concepts be applied to learning and teaching? It is important to note that efficiency and effectiveness are not completely correlated. Sometimes the need to operate efficiently might cause us to be less effective, for example when the last few percent of effectiveness demand an extraordinary amount of time thus diminishing our efficiency. Often in teaching, and sometimes in learning too, we have to make compromises between effectiveness and efficiency.

Efficiency and effectiveness can be applied to all aspects of the teacher's role; for example all teachers undertake substantial amounts of administration. Gains in efficiency in one area, perhaps due to the use of computer tools, may free up time leading to greater effectiveness elsewhere. To be able to work efficiently, time spent on administration and supporting the learning environment should be minimised.

\subsection{Usability}

There is a considerable disincentive to use software systems that are slow to learn and/or hard to use, and conversely systems that are easy to learn and use tend to enhance our productivity, provided they are effective. These considerations point us towards systems that are simple to learn, intuitive to operate, and easy to maintain.

Similar considerations to the above apply to the learners who use software as a means to an end; the end is successful learning, the tools used should not distract learners because they are not entirely effective, or hard to learn, or hard to use. Students should not spend more time on managing their learning infrastructure than they do on carrying out learning tasks. However there are trade-offs here as 
well. Some software can be hard to learn, but easy to use (once learned) and in such cases there is justification for the extra effort needed to learn the software in order to promote effective learning of subject matter in the future.

\subsection{The web as a medium}

The world wide web is simply one medium that a teacher can use to structure learning materials, and this might be done well, where students work actively in a student-centred environment, including opportunities to interact with the teacher through forms, forums and other communication software, or poorly, where students receive content delivered from the server, with no opportunity to interact with the teacher. It is quite natural in web-based eLearning to structure the environment, and provide multiple paths through it, and this encourages students to become more active in their learning by accessing the environment in ways that suit their own learning style.

Do we need the 'e' in 'e-Learning'? An answer to this question is "only if we need the 'b' in 'bookbased learning' or the 't' in 'tutorial-based learning'”. In other words the learning is more important than the medium used to facilitate the learning. Some might say that the term 'e-Learning' is an unfortunate contraction that muddles the medium with the message.

As computing professionals we are constantly aware of the distinction between software tools and the data or content that our tools store and manipulate, and while we enjoy using and adapting the tools in interesting ways, our ultimate goal in eLearning is helping our students to learn.

\section{4. e-Learning}

e-Learning appears to be moving in a direction that is much more informal, web-centric and employing a variety of widely used tools. New tools are adopted as they become available; one such example is the use of twitter in education, discussed below. Old tools become superseded by something better. [1] claims that "YouTube is developing into a kind of University of the Grassroots". It is our view that the days of the closed, corporate VLE are numbered as the sole medium for e-Learning. Universities, and other learning organisations, will begin to embrace a much more lightweight, free, open source, heterogeneous set of tools to facilitate student learning. The development of 'cloud computing' pushes organisations in the same direction of utilising third party systems and tools with the data stored on the third party servers; in such an environment the kind of e-Learning we are advocating will thrive.

There have been numerous studies of the effectiveness of e-Learning. Most of these take the form of comparing the learning achieved in a conventional teaching regime, with the same learning conducted in an e-Learning environment. [2] found that "video-driven multimedia, web-based instruction was not only pedagogically equivalent in terms of knowledge gains to the live instruction but that the knowledge gains were slightly higher among the web-based participants". [3] found that "effects compared with non-Internet instructional methods are heterogeneous and generally small, suggesting effectiveness similar to traditional methods." According to [4] "The results of the study indicated that the traditional and distance students did not differ significantly in their performance on graded assignments... No significant differences were found between the traditional and distance students in mean scores on the five tests or the two papers." Similarly [5] found no significant difference between learners in web-based and classroom environments: "while the online group scored slightly better that the campus group on the class posttest, the difference in performance was not statistically significant."

Some studies indicate better performance in a web-based learning environment, while others find the traditional classroom environment yields significantly better learning (as measured by performance in post-learning tests); however perhaps the majority of these frequently conducted experiments have found no significant difference in the learning achieved by technologically mediated and traditionally mediated groups. Our tentative conclusion based on these meta-studies is that the effectiveness of e-Learning is sufficiently close to that of traditional classroom learning as to make no difference, whichever method is used.

If we therefore put effectiveness to one side, are there any differences in efficiency when comparing e-Learning with traditional methods? As indicated above, 'efficiency' is to do with the number of errors in a process, and with the speed at which tasks may be accomplished. We need to consider the efficiency for the teachers, and for the learners.

A University teacher's time is usually split among four main tasks: teaching; research and other scholarly activities; the assessment of students; and administration. Can electronic methods offer productivity gains in the teaching dimension, or the assessment dimension of a teacher's work? Computer systems have been used to support administration since the 1950s; can e-administration enable a teacher to operate more efficiently, thus freeing time to focus on other aspects of their work? Or is the setting up of computer-based learning, and the ongoing maintenance of the resources, so timeconsuming that it acts as a distraction, making the teacher more inefficient? We consider some case studies from our own work, and some third party case studies. 
How does the teacher's role change when developing e-Learning? It depends on the learners, the environment and the kind of learning desired. If students have regular, timetabled meetings with teachers in an educational institution the role of eLearning tends to be as a supplement to conventional learning, while for distance learners e-Learning is a substitute.

How does the learner's role change in an eLearning environment? Learners who are used to a highly didactic, teacher-centred approach following a non-divergent sequence of listening, reading and doing exercises prescribed by the teacher may have difficulty in adjusting to a more student-centred environment where they have to make choices among a variety of routes to learning, where they need to be more pro-active and experimental in discovering what is most efficient and effective for them personally in achieving successful learning.

We teach undergraduate and graduate students within a University and our use of e-Learning is mainly as a supplement to conventional methods. What effect does this development have on our effectiveness and our efficiency?

\section{Main Characteristics of effective and efficient e-Learning tools}

Important principles, derived from our experience working with students and colleagues, include 'simplicity', 'versatility', 'decentralisation' and 'openness'. These are the characteristics of web tools that seem to us to be highly desirable because while the majority of teachers can operate computers, and in particular web browsers, without significant problems, not all teachers have significant expertise in the more technical aspects of computer systems such as computer programming, or setting up networks, etc. Simple tools such as shared bookmarks, a shared website for learning, blogging software applied to learning, or an assessment management system can all be developed easily and relatively independently, but when combined together they provide a useful resource. We are committed to the idea that good ideas need not be complicated.

As computing professionals, reuse of code and/or electronic material is a common practice. Using simple, open systems enables us to apply these principles to our teaching material. When teaching, some content and material can overlap several courses, hence the need to be able to "share" material. VLEs are designed to create independent courses. Course documents (lectures, exercises, etc.) are therefore attached to one course only. The capacity (or lack of) to reuse material is an issue for teachers, since dealing with duplicate files is a timeconsuming and error-prone task. Learning materials often need to be updated quite regularly. This maintenance of resources is very much more efficient if a single update is propagated automatically across all the courses using those resources. The internet itself, with the ability to easily link documents (as many times as we want) enables re-use of teaching material in a very simple way.

The internet, and the world wide web were designed to be robust, versatile, decentralised and open. The medium makes it natural to combine webbased systems. We are not seeking to provide an allin-one, complete solution to the "problem" of teaching; our approach is to develop useful tools that support particular tasks, and then loosely combine these sub-systems. This approach works well in our situation where the majority of our students are oncampus, working in a blended learning environment.

Another important advantage of loosely coupled, open systems is the flexibility afforded by being able to incorporate useful new developments as they appear (or to integrate custom-designed developments). There is however an obverse side to this advantage when third party software disappears. For example we have found bookmark sharing using the del.icio.us website a useful facility in encouraging students to read more widely, and to share the results of their reading with others (see below). In December 2010 there was an announcement that Yahoo! would no longer support del.icio.us and consequently we were faced with the prospect of a useful tool disappearing. Yahoo! has subsequently announced that the software will continue in some form, although no longer as part of the company [6]. How does our system withstand such shocks?

The issue here concerns the security for the teacher and the student in depending on third party software. Are there always good alternatives when a component part of the learning system vanishes? In the case of del.icio.us there are suitable alternatives, in part due to other organisations copying the success of del.icio.us. In general we believe that there is sufficient redundancy in web applications to make it unlikely that there will be no alternative to a particular sub-system available, although there may sometimes be some loss of quality. If there is no good alternative to a sub-system that vanishes we have the ability to write software that may address the loss, although such work diminishes our efficiency as teachers due to the time required.

What should a University do when the continuity of the infrastructure cannot be guaranteed? As teachers, we may not see this as a significant problem, but University managers may have a different perspective, arguing that the University is the warrant of the service provided to students. Some Information System managers also view the use of third party tools a bad choice because if, instead of asking the University to provide some reliable 
services (such as online storage, etc.), a teacher uses third party software, the teacher's needs go unnoticed and therefore IS managers have no evidence of resource utilisation to back up their own requests for resources. Another concern is privacy related. The use of Internet tools as teaching tools usually implies that the data will be either public, or stored privately on a third party server that is controlled by the third party. Some argue that what happens in a course should not be public [7], similarly to what happens in the classroom is not out in the open, nor should data (and especially personally identifiable data) be stored in a place that cannot be guaranteed secure. These concerns are particularly sharp when using social networking tools.

Finally, there is a related issue of "branding" where a University might wish to be able to present a VLE as wholly owned and maintained by the University, rather than present the learning environment as a collection of third party tools ... in the latter case students may begin to question what exactly they are buying when they pay their University fees if all they see are free tools available to the general web-public. To address this issue there have been some attempts to incorporate third party software through their published APIs into a "University branded" environment that is more unified, [8]. For students, and teachers, of Computing this is not a significant problem because we expect such students to learn about many different flavours of software and how they might be combined; other students however may prefer to have the combining done for them along the lines indicated by [8].

\section{Case studies}

\section{1. e-Assessment}

An assessment system was developed by the Département d'Informatique at the Université de Caen, France, to store, view, explore, annotate and grade students' work. For a typical assessment, students usually need to create a set of programs and upload an archive into the system. Since an archive can be made up of many files, assessment reviewing, annotating and grading can turn into an overwhelming file management task, exploring archives and files. To avoid such time consuming tasks, a web interface was created for teachers. Annotations can be made on the assessment level or on a specific file, and private annotations (ie. visible only by the teacher) are also available. Intensive use of Ajax technology enables the user to interact with the interface without having to reload the whole page, as shown Figure 1.

At the end of the grading work, teachers can use a mailing tool to automatically email each student his or her grade along with all the annotations. The system can also be used without giving grades, so as to use it for feedback only.

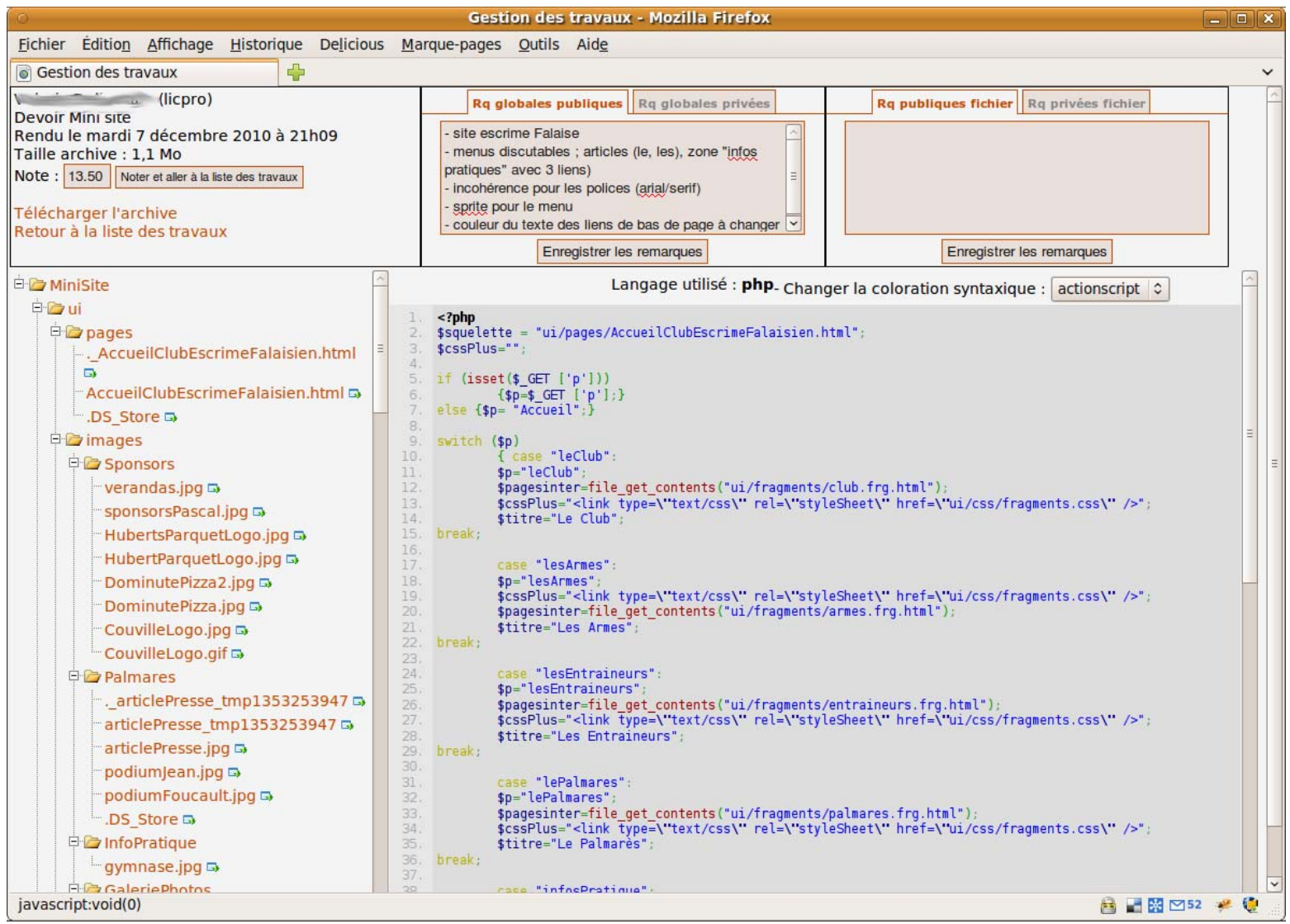

Figure 1. Assessment system at the Département d'Informatique 
Users' feedback has been very positive : many teachers like the fact that they don't receive the students work in their email any more, and that they don't need to manipulate any files and can annotate as they view files. It is interesting to note that none of the teachers used the private annotation functionality. Several teachers said they particularly like the simplicity and ease of use of the system, and that it enables them to easily give feedback to the students via the comment system. Students like to have a placeholder for their assessment and can easily upload their work. Similarly it provides teachers with a unique space to view their students' work and makes it very quick to go back and check one's work when a student asks for more information about his grade and/or the remarks made. This is far more efficient than handling student work within a general purpose email system for example.

After being used for almost 3 years, the system proves to be very efficient to review a complete set of programs developed by students. Effectiveness has been improved with the possibility to view different works in a few clicks, and to annotate students' work without switching between applications or editors. Future work would need to improve assessment lists displays, add a statistics module and enable group work where an assessment is uploaded by a group of students instead of an individual.

A completely separate project has been conducted by a group of $\mathrm{PhD}$ students in Caen. They have developed a tool to compare sets of programs in order to detect similarities and possible plagiarism among the work submitted by students [9], [10]. Using a simple open architecture had made possible to connect the plagiarism tool with the assessment system quite easily. Even though more work needs to improve the User Interface so that a teacher will be able to detect if some works are too similar with a couple of clicks, a proof-of-concept has shown that the tools can interconnect.

A student assessment problem at Portsmouth involves dealing with very large groups who are assessed by multiple tutors. We have developed a web-based tool to manage the assessment, and help tutors to rapidly compare the standard of their marking with that of other tutors. The same system enables students to obtain their results simply by pushing a button.

The form of the assessment is a ten minute verbal presentation by students to a tutor where the tutor questions students about their coursework and records the quality of the work and their answers through ten criteria that are listed on a data capture form. Note that tutors are required to judge only the quality of the student's work by grading their work on each of ten criteria as objectively as possible. Transforming these qualitative judgments into a quantitative mark is performed later by software. The

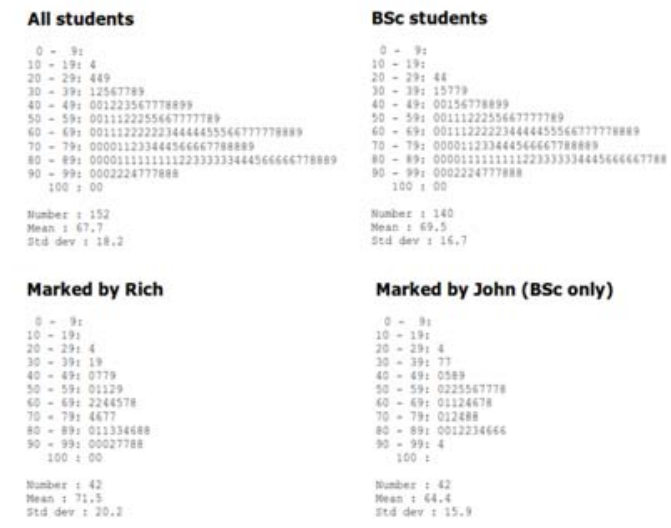

\section{Figure 2. Stem and leaf graph examples}

grades on these paper forms are copied to a web form; when the Submit button is pressed the data is validated, and the grades are converted to numbers which are added together to give an overall mark for the student. Results are recorded in a file and various reports can be obtained, including a number of Stem and Leaf graphs that provide graphical views of all the results, or subsets filtered according to various criteria such as the name of the group the students belong to, or the name of the tutor who did the marking. The latter report is very useful in helping tutors check that their assessing is of a similar standard to those of the other assessors. The stem and leaf graphs shown in Figure 2 are arranged using deciles as the stem, and the units digit of the student mark as the leaf; this provides a convenient view of the results with no loss of information. Students are able to obtain their results from the system simply by viewing an access controlled page so that the student view is restricted to their own result only. The result comprises the mark computed by the system, and any comment that the tutor has entered on the web form.

The software behind this system appears to be reliable; however error is still possible due to the human element, and in particular the transcription of the grades from the paper data capture form into the web form. Fortunately errors are few, at around 2 in 1000 transfers. Typical errors are a transposition error where a student ID code is incorrectly transcribed, resulting in the student seeing no mark, or worse still when a transposition error results in a valid, but incorrect, ID, potentially replacing the results of another student. (This latter error has never occurred, but remains a worrying theoretical possibility.) The software includes some validation code to help mitigate errors, but more development work remains to be done in this area.

Three years experience with this assessment management system leads us to the conclusions that:

(i) decoupling the grading of student work from the marks awarded is a good thing, as it enables the assessor to focus on the student work and how well it satisfies the relevant criteria, freeing them from the 
additional burden of translating their assessment into a quantitative mark;

(ii) the rapid presentation of the results in graphical form is helpful in enabling an assessor to calibrate their judgements against those of other assessors, thus helping to reduce the need for remarking the work of an assessor whose assessments are significantly aberrant;

(iii) students like the early view of their provisional mark; and staff like the fact that distributing marks to students is entirely automated, leaving them nothing to do (very efficient);

(iv) the automatic generation of stem and leaf graphs enhances efficiency by facilitating assessment administration;

(v) the software carries out checks, such as all fields completed, that might otherwise have to be done manually; this is clearly efficient;

(vi) the system appears to be relatively errorfree; this is efficient compared with systems that require mistakes to be corrected; error tracking is enabled by retaining the paper data capture forms, but the need to use these has been very rare.

Our overall conclusion is that the assessment management system described here makes a positive contribution to the efficiency of up to five assessors in any one presentation of the course. The system is simple enough for new assessors to learn to operate it immediately. The system is specific to the particular form of assessment although some of the general features such as translating grades into marks, and rewriting the marks as stem and leaf graphs are quite general and easily transfer to other automated assessment systems. In overall terms the payback for the development time is in credit after three years use. The software itself was quick to write, most of the time and effort were spent in designing a system that was simple and robust. Given the large cohorts to be assessed the work is conducted under considerable pressure, and a highly efficient operation is crucial to the success of the assessment overall. In terms of efficiency the system is a success.

We have a high degree of confidence that the best students get the highest marks, and vice versa. The assessment by viva voce, even though it is only ten minutes long, is effective in identifying students who

This week you will learn some basic html markup, and how to serve web pages from your own server.

2:56 PM 0 ct 11th, 2009 from

New faq posted at http://userweb.port.ac.uk/ rosbotto

/webauh/current/assessment.php\#fac

3:34 AM Dec 8th, 2009 from web

penhart @webaud If anyone from Mon 9am/10am tutorials still needs assessment, Penny's office is BK1.27. Or definitely come to next week's tutorials.

Figure 3. Notices on webaud twitter stream are not able to explain their work, and this has a considerable deterrent effect on students plagiarising the work of others. Hence in terms of effectiveness the system seems successful.

The decentralised nature of the software enables tutors to work in parallel on the system, combined with the calculations and data visualisation performed by the software, the efficiency obtained makes the software essential in achieving rapid, reliable assessment.

\subsection{Web tools applied to learning}

The explosion of the Web2.0 has led to a profusion of sites offering a variety of services, usually free of charge. In our teaching practice we have mostly used twitter and delicious, which provide high quality ready-to-use tools, but we also developed our own tools with simplicity of use in mind. As noted in other studies, using web 2.0 tools can be time consuming, particularly if they are used without self-discipline, but students recognise and appreciate the teacher's work and view it as a motivating factor [11].

4.2.1. twitter. twitter is a micro-blogging tool where posts, limited to 140 characters, are posted to a public server and other twitter users may "follow" the posts of other users. The software seemed an ideal application as a channel for short notices to students about a unit of study. A unit noticeboard was set up [12] and four tutors working on the unit

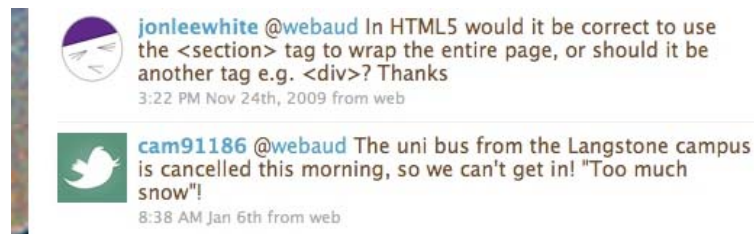

Figure 4. Questions on webaud twitter stream

used the twitter stream to post notices to students.

It should be noted that the web authoring and design (webaud) unit of study is for first year undergraduates and has about 200 registered students... so communication can be a real problem!

Very quickly the students, of their own volition, started to use the twitter stream to ask questions to tutors, and to share information among themselves.

This seemed an entirely natural thing to do. Some examples are Notices (as shown Figure 3); Questions (see Figure 4 - note that the second of these is not really a question, but an example of a student using twitter as a noticeboard to inform tutors); and Discussion (see Figure 5). 
\begin{tabular}{l} 
RUE \\
THE \\
NHROR \\
\hline
\end{tabular}

ShaneLF @webaud I found a brilliant webpage for using wordpress with xampp: http://tamba2.org.uk/wordpress xampp/ $\wedge \wedge{ }^{*}$ creds!* 1.52 AM Oct 16th, 200

\section{Figure 5. Discussions on webaud twitter stream}

It was necessary to adapt the standard twitter mode of operation to the learning situation, but such adaptation was slight. For example in the bio section of the twitter account the following was posted: "webaud is a course, not a human being. Followers are participating students. Webaud follows nobody!” The point here was to reassure students that while they could use an existing twitter account to follow the staff notices posted to the twitter account, the University twitter entity would respect their privacy and not follow their postings from their existing account. Another management issue relates to followers who are not students on the unit of study, many of these are robot entities designed to gather intelligence and post material to target twitter feeds. It is a minor matter to remove these unwanted followers from time to time.

Given the context of a course with a large number of students (typically around 200), many of whom had only limited IT skills, the twitter stream proved to be very effective as a way of communicating with students because the students had no difficulty in setting up their own twitter IDs and following the notices posted on the unit twitter stream. The strengths of the system were the very low overheads in getting involved, the ease of use and the integration (through standard web links) to items in the unit website. A significant bonus strength was the way the twitter stream functioned as a two-way street with students posting their own notices and questions; this seems a significant advantage over alternative methods such as the (one-way only) use of RSS to post notices. In conclusion twitter has proved to be very effective as a noticeboard with added value.

In terms of efficiency we can compare operating the twitter noticeboard with a physical noticeboard. Twitter notices are text only (although they may link to an image); some might see the need to click through to an image to be inefficient. However the overwhelming efficiency gain in using the twitter noticeboard compared with a conventional noticeboard in a fixed place is that the twitter noticeboard is online and visible in any place where the students have an Internet connection. A good example is the notice posted by a student about the failure of the University bus, even though he was stuck in a remote location, the noticeboard was still accessible. The time to reach the twitter noticeboard is significantly less than the time needed to travel to a physical noticeboard, and this represents a huge efficiency gain.

4.2.2. delicious. Delicious is a social bookmarking site that allows you to store, share and discover bookmarks (ie. links to sites you like). Each bookmark is associated with tags as a means of classification. Many widgets can now display delicious bookmarks with the use of RSS feeds. Using a public system to store bookmarks enables a teacher to re-use the resources instead of re-creating them or copy/paste them. A Firefox extension enables a user to save bookmarks and suggests tags to use based on what tags previous delicious users have chosen.

Delicious RSS feeds are used to share bookmarks with other applications, such as a blog. Wordpress for example includes a delicious widget that enables a blogger to publish his bookmarks within minutes, with the possibility to select bookmarks to display thanks to the tags associated. In order to easily identify teachers and students bookmarks, specific tags were created (etu.info.unicaen.web for students and pedago.unicaen for teachers). Figure 6 shows the latest activity associated with that tag.

The system proves very efficient and easy to use, enabling teachers to quickly share resources and find links to include in lecture references. Six months after starting the experiment, results are mixed Even though numerous bookmarks have been added, only a few students account for most of the activity. It is important to note that these students are also the ones that make most use of Web2.0 tools such as twitter, delicious, etc.

Another lesson can be learned from this

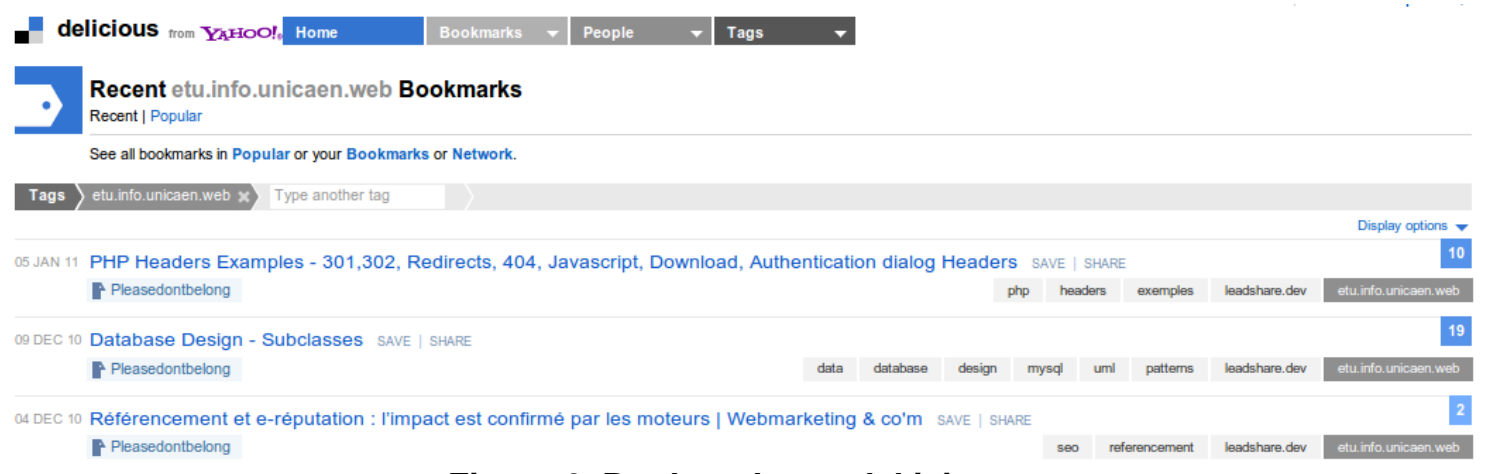

Figure 6. Bookmarks on del.icio.us 
experience. Use of delicious was optional for students and was there to enhance their learning experience and share resources. There was no learning task or assessment associated. Hence most students have not invested much of their time into the tools offered. This rather disappointing phenomenon suggests a preference by some, perhaps most, students for their learning to be teacher-led. This may be a consequence of their previous experiences in school and college, combined with a tendency among many to be passive rather than active in their learning. Many students want to learn in an efficient way, avoiding unnecessary work, and they trust the teacher to lead them along an efficient and effective route, perhaps not understanding the large variability in personal learning needs. We are still a long way from the more individualised, student-centred learning environment that has been advocated for many years by educational experts; the standard teacher-centred approach to mass education is remarkably robust, despite the many students who fail to realise their full potential. Teachers naturally have a better understanding of a subject than do students and this can easily lead to students deferring to the teacher's perceived superior knowledge and becoming passive learners. Learning is naturally hard work, and students need confidence to admit that their understanding is confused, or incomplete. Helping students handle the learning process with more self-confidence is part of the role of any good teacher. e-Learning can help decouple learning from the teacher, and hence reduce dependence on the teacher, by introducing additional resources that are under the control of the student, thus encouraging students to become more experimental in their learning, as was the delicious experiment designed for. Students do not always realize that if the common knowledge of the group improves, then it will benefit each learning individual.

This final point is important. Although not every student appears to take advantage of the potential for shared learning, the process does appear to be one of the few examples where student learning can become more effective (rather than more efficient). If learning is perceived as a shared, group process, rather than a competitive, individualised one, then learning is enhanced by combining together small individual contributions into a shared research environment. Benefit derives as much from the active participation of making discoveries as it does from the following of the contributions of others, resulting in a better (more effective) kind of learning.

4.2.3. The unit website. For all of us the most important part of an e-Learning infrastructure is the website for a unit of study. The website acts as the hub, or meeting point, from which links to other tools, and paths through the learning can be explored.
At Portsmouth there is a VLE that all students use as a portal to learning resources, most of which are within the VLE itself. However there are problems. Teachers complain about the intricacy of setting up learning materials and maintaining them within the VLE. Another problem is that the proprietary VLE uses sub-systems that parallel commonly used resources such as email and instant messaging; however the VLE resources are frequently perceived as less effective and less satisfying to use than their everyday equivalents. A good deal of the intricacy derives from the need to guard the resources from illegal copying; for example simple web pages are transformed into large chunks of relatively impenetrable javascript. Essentially the VLE acts as a database that stores and retrieves web pages in an unusual, proprietary fashion. This makes a considerable problem for teachers who wish to teach web programming by example. The VLE is unsatisfactory for such usage. Our standard practice is to create a portal page within the VLE that links to the true unit website that lies in a separate domain, using a standard web server. For example Adtis' homepage is a very simple html web page that is easy to update from year to year acting as the root of the true unit website. Navigation provides access to the twitter stream used as a unit noticeboard (for adtis); links to third party resources that promote independent learning; a table that provides links to all the timetabled lectures and tutorial sessions; the assessment used in the unit; and finally a narrative description that provides an integrating overview of the whole unit. These elements, or web pages, are sufficient to enable students to participate in the unit of study in a student-centred way. In the case of Portsmouth, some units that are taught wholly by distance learning have exactly the same structure in the unit website, the only change needed is to transform some of the lectures and tutorials so that they are able to function independently of any face to face contact with the teacher.

The same scenario happens at the University of Caen, and teachers involved in web programming have set up a specific site to publish content for students. In both cases, websites have a standard structure with a simple navigation that gives an overview of the whole course with links to the relevant learning materials. At the Département d'Informatique, the pages are run by several teachers who share their resources. Re-use of teaching material is highly encouraged within the team and content can very easily be shared between teachers and classes thanks to a flexible architecture. Students know that they can find resources used for other courses. Content is published with a Creative Commons license and is encouraged to be shared with the world through "Sharing" links to twitter, facebook and delicious.

The system enhances effectiveness of learning by 
encouraging students to find their own way through the unit resources, in a way that matches their preexisting knowledge and their preferred learning style. The system also makes the teacher's work more efficient because students are enabled to work independently without close instruction by the teacher; these gains are of course magnified when teaching large groups. These efficiency gains have to be offset against the effort required to set up the system. In this case all the web pages are designed to be simple and hence easy to maintain. However the strategy adopted by [13] seems even more efficient. David Wiley uses WordPress, a standard software used by bloggers worldwide, as an interface to his unit of study on open courseware.

From an efficiency point of view, Wiley's use of powerful software makes it easy to maintain and reproduce the front-end to his learning resources. Clearly operating in this way requires the teacher to be able to create and maintain simple web pages, or following Wiley to use WordPress for the same purpose. With little experience or computer knowledge, a Wordpress blog can be set up very quickly. For example, to manage a workshop at the Institut d'Administration des Entreprises (Caen, France) aimed at helping students discover web based tools such as blogs, wikis, social bookmarking, etc., a Wordpress blog was set up within one hour, providing a few articles and links and allowing students to experiment live while the workshop was taking place.

These skills in writing web pages are comparatively simple, and will become more and more common among teachers as a more web-literate generation of teachers enter the profession; but in any case the skills are not difficult for anybody to learn, as evidenced by the thousands of self-taught bloggers throughout the world.

\section{Conclusions}

The effectiveness of e-Learning is determined not by the medium but by the learning design. Different learning media have different strengths, but software is not the "killer medium" that will supercede all others. The priority of design over medium is true for conventional paper-based learning with oral instruction, or learning through a text book, as well as for learning through software. While it is always fun to play with new toys, we should not allow the 'e' to dominate the 'learning'.

Factors that relate to the effectiveness and efficiency of e-Learning include: class size; IT skills of the teacher/developer; the development overheads of e-Learning compared with conventional learning; maintenance overheads of e-Learning compared with conventional learning (preparing for future editions of the course); volatility of the learning domain (high volatility implies high maintenance); and the reusability of e-Learning resources.

As class size increases, the relationship between students and teacher becomes more remote, and the students naturally tend towards becoming distance learners. Hence as class size grows e-Learning becomes more valuable as a supplement in support of face to face lectures. Also large classes tend to mean there is always a fraction of absent students for legitimate or illegitimate reasons. Again independent e-Learning helps those students who miss classes to catch up. We have also seen that e-Learning tools can help improve the efficiency of teachers when working with large groups of students.

The IT skills of the teacher make a difference to how efficient that teacher is in developing and maintaining an e-Learning infrastructure. The issue here is how naturally a teacher writes a web page, or a blog, including links to other resources. This is a style of writing that improves with practice. As more 'digital natives' become teachers we can expect to see teachers writing websites as naturally as they wrote books and other learning resources in the twentieth century. The development and maintenance of eLearning resources is a related matter, and these problems are not really very different from the same issues in conventional paper-based learning that involves extensive preparation and reproduction of resources in advance of teaching. It isn't clear whether e-Learning is more or less efficient than conventional learning in this respect. Teachers have always attempted to design their resources so that they may be reused in other learning situations. e-Learning has considerable advantages over conventional methods simply because software is a much more tractable medium than paper. In this respect the efficiency of software based resources is supreme for the same reason that word processors have replaced typewriters.

In this paper we have reviewed several tools, developed by individuals or small groups, that enhance both the efficiency and effectiveness of teachers in their teaching practice or assessment and administration tasks, and promote more effective and more efficient learning for students. We show that small applications that are very flexible and easy to adapt to different situations can greatly reduce time spent on tasks, and can promote efficient learning among our students.

\section{References}

[1] Keegan. V. (December 2009 ) 'Youtube: the people's university of the internet', The Guardian; http://www.guardian.co.uk/technology/2009/dec/02/youtub e-peoples-university-internet (24 January 2011)

[2] Pang., K. (2009) ' Video-driven multimedia, webbased training in the corporat sector: Pedagogical equivalence and component effectiveness', The 
International Review of Research in Open and Distance Learning, 10(3).

[3] Cook, D. A. and Levinson, A. J. and Garside, S. and Dupras, D. M. and Erwin, P. J. and Montori, V. M. (September 2008) 'Internet-based learning in the health professions', Journal of the American Medical Association, 300(10):1181-1196.

[4] Smith, R. J. andPalm, L. J. (2007) 'Comparing learning outcomes between traditional and distance introduction to philosophy courses' Discourse: Learning and Teaching in Philosophy and Religious Studies, 6(2):205-226.

[5] Thirunarayanan, M. and Perez-Prad, A. (2001). 'Comparing web-based and classroom-based learning: A quantitative study', Journal of Research on Computing in Education, 34(2):131-137.

[6] Delicious Blog (2010) 'What's next for delicious?' http://blog.delicious.com/blog/2010/12/whats-next-fordelicious.html (15 January 2011)

[7] Ong, C. and Lai, Y. and Wang, Y (July 2004), 'Factors affecting engineers' acceptance of asynchronous e-learning systems in high-tech companies', Information \& Management, 41 (6), pp. 795-804.

[8] Wilson, S. and Liber, O. and Johnson, M. and Beauvoir, P. and Sharples, P. and Milligan, C. (June2007), 'Personal Learning Environments: Challenging the dominant design of educational systems', Journal of eLearning and Knowledge Society, 3 (2), pp.27-38.

[9] Lesner, B. and Brixtel, R. and Bagan, G. and Bazin, C. (2010), 'A Novel Framework to Detect Source Code Plagiarism: Now, Students Have to Work for Real!', in Proceedings of the 2010 ACM SAC'10 ,p.57-58.

[10] Robbes, R. and Brixtel, R. and Fontaine, M. and Lesner, B. and Bazin, B. (2010), 'Language-Independent Clone Detection Applied to Plagiarism Detection', in Proceedings of the Tenth IEEE International Working Conference on Source Code Analysis and Manipulation, IEEE Computer Society.

[11] Blees, I. and Rittberger, M. (June 2009), 'Web 2.0 learning environment: Concept, implementation, evaluation', eLearning Papers,

http://www.elearningeuropa.info/files/media/media19743. pdf (24 January 2011)

[12] Rosbottom, J. (2010), 'Unit noticeboard' http://twitter.com/webaud (9 April 2010)

[13] Wiley, D. (2010) 'Blogs, wikis, and new media, an experiment in republishing opencourseware.', http://newmediaocw.wordpress.com, (24 January 2011) 